\title{
Formal Specification and Model Checking of A* Algorithm
}

\author{
Kazuhiro Ogata \\ School of Information Science \\ Japan Advanced Institute of Science and Technology (JAIST) \\ 1-1 Asahidai, Nomi, Ishikawa 923-1292, Japan \\ Email:ogata@jaist.ac.jp
}

\begin{abstract}
A* algorithm is formally specified in Maude and model checked with the Maude LTL model checker. We take into account a graph such that it is a DAG, a goal node is reachable from a start node and each edge is given a nonnegative weight. If $h$ is admissible, namely that $h(n)$ never overestimates the cost to the goal from $n$ for all nodes $n$, then $A^{*}$ finds a shortest path. The condition, however, can be relaxed. Our model checking experiments make us conjecture that if there exists a shortest path such that for each node $n$ in the path $\mathrm{h}(n)$ plus the cost to $n$ from the start node is less than the cost of any non-shortest path to the goal from the start, $A^{*}$ finds a shortest path.
\end{abstract}

Keywords-A* algorithm; Dijkstra algorithm; LTL; Maude; Model checking

\section{INTRODUCTION}

$A^{*}$ algorithm [1] is a generalized version of Dijkstra (shortest path finding) algorithm [2]. It uses an estimation of the distance between the goal node and each of the edge nodes to select the next node to be tackled. Dijkstra algorithm treats the estimation as 0 .

$\mathrm{A}^{*}$ is formally specified in Maude [3], a rewriting logicbased specification/programming language equipped with many facilities, among which are model checking ones (a reachability analyzer and an LTL model checker). We model check with the LTL model checker that $\mathrm{A}^{*}$ terminates and finds a shortest path. Our model checking experiments say that $\mathrm{A}^{*}$ always halts and if the estimation $\mathrm{h}$ is admissible, namely that $\mathrm{h}(n)$ never overestimates the cost to the goal from $n$ for all nodes $n$, then $\mathrm{A}^{*}$ finds a shortest path. The condition, however, can be relaxed. Our model checking experiments make us conjecture that if there exists a shortest path such that for each node $n$ in the path $\mathrm{h}(n)$ plus the cost to $n$ from the start node is less than the cost of any nonshortest path to the goal from the start, $A^{*}$ finds a shortest path.

The contribution of the work described in the present paper is to demonstrate how $\mathrm{A}^{*}$ is formally specified in Maude and model checked with the Maude LTL model checker and to find a relaxed sufficient condition, through model checking experiments, that $\mathrm{A}^{*}$ finds a shortest path.

This work was partially supported by JSPS KAKENHI Grant Number JP26240008 \& JP19H04082.

DOI reference number: 10.18293/SEKE2019-022
Saberi, Groote and Keshishzadeh [4] have formally specified in the mCRL2 language a simple path planning algorithm that makes multiple robots reach a destination on a discretized planar surface, described properties in $\mu$ calculus and conducted model checking experiments that the algorithm enjoys the properties. The desired properties they have taken into account are deadlock-freeness, collisionfreeness and reachability. Their motivation to conduct the research is to demonstrate how useful model checking is to verify that the collective behavior of multiple robots satisfy some desired properties. Maragos, Kleftouris and Ziogou [5] have formally modeled a path finding planning in Colored Petri Nets (CP-Nets). Given a discretized planar space where there is one robot located in one position, there are some obstacles located in some positions and there is a goal position, the planning is to find a path along which the robot reaches the goal. Their CP-Nets model is executable with the Design/CPN tool. Their main motivation to conduct the research is to demonstrate how powerful CP-Nets are for simulating such a planning. Alphan, Smith, Belta and Rus [6] have proposed a method that automatically plans optimal paths for a group of robots that satisfy LTL properties. They formally modeled as a timed automaton a system in which multiple robots asynchronously move to some vertexes from the current vertexes on a graph if there are direct edges from the latter to the former. They use a bi-simulation relation between a timed automaton that is inherently infinite and a finite-state transition system so that they can use their earlier algorithm dedicated to a single-robot system.

The rest of the paper is organized as follows: $\S$ II Preliminaries, § III A* Algorithm, $§$ IV Formal Specification, $\S \mathrm{V}$ Model Checking, and $\S$ VI Conclusion.

\section{PRELIMINARIES}

A Kripke structure $K$ is $\langle S, I, T, P, L\rangle$, where $S$ is a set of states, $I \subseteq S$ is the set of initial states, $T \subseteq S \times S$ is a total binary relation over $S, P$ is a set of atomic propositions and $L$ is a labeling function whose type is $S \rightarrow 2^{P}$. Each element $\left(s, s^{\prime}\right) \in T$ is called a state transition from $s$ to $s^{\prime}$ and $T$ may be called the state transitions (with respect to $K$ ). For a state $s \in S, L(s)$ is the set of atomic propositions that hold in $s$. A path $\pi$ is an infinite sequence $s_{0}, \ldots, s_{i}, s_{i+1}, \ldots$ of states such that $s_{i} \in S$ and 
$\left(s_{i}, s_{i+1}\right) \in T$ for each $i$. Let $\pi^{i}$ be $s_{i}, s_{i+1}, \ldots$ and $\pi(i)$ be $s_{i}$. Let $P$ be the set of all paths. $\pi$ is called a computation if $\pi(0) \in I$. Let $C$ be the set of all computations.

The syntax of a formula $\varphi$ in LTL for $K$ is $\varphi::=$ $\top|p| \neg \varphi|\varphi \wedge \varphi| \bigcirc \varphi \mid \varphi \mathcal{U} \varphi$, where $p \in P$. Let $\mathcal{F}$ be the set of all formulas in LTL for $K$. An arbitrary path $\pi \in \mathcal{P}$ of $K$ and an arbitrary LTL formula $\varphi \in \mathcal{F}$ of $K$, $K, \pi \models \varphi$ is inductively defined as $K, \pi \models \top, K, \pi \models p$ iff $p \in L(\pi(0)), K, \pi \models \neg \varphi_{1}$ iff $K, \pi \not \models \varphi_{1}, K, \pi \models \varphi_{1} \wedge \varphi_{2}$ iff $K, \pi \models \varphi_{1}$ and $K, \pi \models \varphi_{2}, K, \pi \models \bigcirc \varphi_{1}$ iff $K, \pi^{1} \models \varphi_{1}$, and $K, \pi \models \varphi_{1} \mathcal{U} \varphi_{2}$ iff there exists a natural number $i$ such that $K, \pi^{i} \models \varphi_{2}$ and for all natural numbers $j<i$, $K, \pi^{j} \models \varphi_{1}$, where $\varphi_{1}$ and $\varphi_{2}$ are LTL formulas. Then, $K \mid=\varphi$ iff $K, \pi \models \varphi$ for each computation $\pi \in \mathcal{C}$ of $K$. The temporal connectives $\bigcirc$ and $\mathcal{U}$ are called the next connective and the until connective, respectively. The other logical and temporal connectives are defined as usual as follows: $\perp \triangleq \neg \top, \varphi_{1} \vee \varphi_{2} \triangleq \neg\left(\neg \varphi_{1} \wedge \neg \varphi_{2}\right), \varphi_{1} \Rightarrow \varphi_{2} \triangleq \neg \varphi_{1} \vee \varphi_{2}$, $\diamond \varphi \triangleq T \mathcal{U} \varphi$, and $\square \varphi \triangleq \neg(\diamond \neg \varphi)$. The temporal connectives $\diamond$ and $\square$ are called the eventually connective and the always connective, respectively.

There are multiple possible ways to express states. We express a state as a braced associative-commutative (AC) collection of name-value pairs. AC collections are called soups, and name-value pairs are called observable components. That is, a state is expressed as a braced soup of observable components. The juxtaposition operator is used as the constructor of soups. Let $o c_{1}, o c_{2}, o c_{3}$ be observable components, and then $o c_{1} O c_{2} O c_{3}$ is the soup of those three observable components. A state is expressed as $\left\{\begin{array}{lll}o c_{1} & o c_{2} & o c_{3}\end{array}\right\}$. There are multiple possible ways to specify state transitions. We specify them as rewrite rules. Concretely, we use Maude [3], a programming/specification language based on rewriting logic. Maude makes it possible to specify complex systems flexibly and is also equipped with model checking facilities (a reachability analyzer and an LTL model checker). A conditional rewrite rule (or just a rule) is in the form $\mathrm{crl}[l b]: l=>\operatorname{if} \ldots / c_{i} \wedge$ $\ldots$, where $l b$ is the label given to the rule and $c_{i}$ is part of the condition, which may be an equation $l c_{i}=r c_{i}$. The negation of $l c_{i}=r c_{i}$ could be written as $\left(l c_{i}=/=r c_{i}\right)=$ true, where $=$ true could be omitted. If the condition $\ldots \wedge c_{i} / \ldots$ holds under some substitution $\sigma, \sigma(l)$ can be replaced with $\sigma(r)$.

The search command tries to find a state reachable from $t$ such that the state matches $p$ and satisfies $c$ :

search [1] in $M: t=>* p$ such that $c$.

where $M$ is a specification of the $S$ and $T$ parts of $K . t$ typically represents an initial state of $K$.

Let init be the only initial state of $K$ and $\varphi$ be an LTL formula. Then, the Maude LTL model checker checks that $K$ satisfies $\varphi$ by reducing modelCheck $($ init,$\varphi)$.

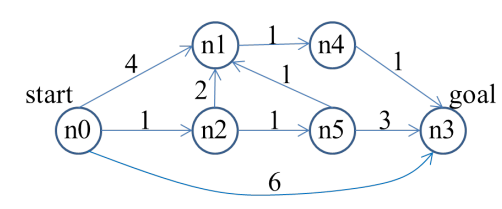

Figure 1. A DAG (1)

\section{A* ALGORITHM}

Let us consider the DAG shown in Fig. 1 in which node $\mathrm{n} 0$ is the start node and node $\mathrm{n} 3$ is the goal node. Let us use the estimation $\mathrm{h}(n)$ for each node $n$ defined as follows: $\mathrm{h}(\mathrm{n} 1)=2, \mathrm{~h}(\mathrm{n} 3)=0$ and $\mathrm{h}(n)=1$ for any other node $n$. Let $\mathrm{w}\left(n, n^{\prime}\right)$ be the weight of the edge from node $n$ to node $n^{\prime}$. For example, $\mathrm{w}(\mathrm{n} 2, \mathrm{n} 1)=2$. For each node $n$, two pieces $\mathrm{p}(n)$ and $\mathrm{d}(n)$ of information are maintained, where $\mathrm{p}(n)$ is the shortest path found so far to node $n$ from the start node at any given moment and $\mathrm{d}(n)$ is its distance. One more piece $o q$ of information is maintained, which is an ordered queue of node \& natural number pairs, where such pairs are stored in increasing order based on their second elements. When a pair $\langle n, d\rangle$ of node $n$ and natural number $d$ is enqueued into $o q$ that contains some other pairs whose second elements are $d,\langle n, d\rangle$ is stored following all those pairs. When $\langle n, d\rangle$ is enqueued into $o q$ that contains $\left\langle n, d^{\prime}\right\rangle$, if $d^{\prime}<d$, then $\langle n, d\rangle$ is stored at the designated place in $o q$ and $\left\langle n, d^{\prime}\right\rangle$ is deleted and otherwise $\langle n, d\rangle$ is not stored in $o q$. For example, let $o q$ consist of $\langle n 0,1\rangle,\langle n 1,1\rangle,\langle n 3,2\rangle$ and $\langle n 2,3\rangle$ in this order, denoted $\langle n 0,1\rangle|\langle n 1,1\rangle|\langle n 3,2\rangle \mid\langle n 2,3\rangle$, and if $\langle n 3,1\rangle$ is put into $o q$, oq is $\langle n 0,1\rangle|\langle n 1,1\rangle|\langle n 3,1\rangle \mid\langle n 2,3\rangle$. We use empq to represent the empty queue and suppose that empq is an identity of the queue constructor $\mid$, namely that $q \mid \mathrm{empq}=q$ and empq $\mid q=q$ for all queues $q$. We also suppose that a single element $e$ is treated as a singleton queue that only consists of $e$. For example, $\langle n 3,1\rangle$ is treated as the singleton ordered queue that only consists of $\langle n 3,1\rangle$. Node $n$ of each $\langle n, d\rangle$ in $o q$ at any moment is called an edge node at that moment in this paper.

Initially, $\mathrm{p}(\mathrm{n} 0)=\mathrm{n} 0$ (which is the path that only consists of $\mathrm{n} 0), \mathrm{p}(n)=\varepsilon$ (the empty path) for any other node $n$, $\mathrm{d}(\mathrm{n} 0)=0, \mathrm{~d}(n)=\infty$ for any other node $n$ and $o q=\langle n 0,1\rangle$ (which is the ordered queue that only consists of $\langle n 0,1\rangle$, where $\mathrm{d}(\mathrm{n} 0)+\mathrm{h}(\mathrm{n} 0)=1)$.

While $o q$ is not empty, the following is repeated. Let $\langle n, d\rangle$ be the top element of oq. If node $n$ is the goal node, $\mathrm{p}(n)$ is the path to be found from the start node to the goal node. Otherwise, for each direct successor node $n^{\prime}$ of node $n$, if $\mathrm{d}(n)+\mathrm{w}\left(n, n^{\prime}\right)<\mathrm{d}\left(n^{\prime}\right), \mathrm{d}\left(n^{\prime}\right)$ is set to $\mathrm{d}(n)+\mathrm{w}\left(n, n^{\prime}\right)$, $\mathrm{p}\left(n^{\prime}\right)$ is set to the path obtained by adding $n^{\prime}$ to $\mathrm{p}(n)$ at the end and $\left\langle n^{\prime}, \mathrm{d}(n)+\mathrm{w}\left(n, n^{\prime}\right)+\mathrm{h}\left(n^{\prime}\right)\right\rangle$ is enqueued into $o q$. The top element is deleted from $o q$.

At some moment, 
- $\mathrm{p}(\mathrm{n} 0)=\mathrm{n} 0 \& \mathrm{~d}(\mathrm{n} 0)=0$

- $\mathrm{p}(\mathrm{n} 1)=(\mathrm{n} 0 \rightarrow \mathrm{n} 1) \& \mathrm{~d}(\mathrm{n} 1)=4$

- $\mathrm{p}(\mathrm{n} 2)=(\mathrm{n} 0 \rightarrow \mathrm{n} 2) \& \mathrm{~d}(\mathrm{n} 2)=1$

- $\mathrm{p}(\mathrm{n} 3)=(\mathrm{n} 0 \rightarrow \mathrm{n} 3) \& \mathrm{~d}(\mathrm{n} 3)=6$

- $\mathrm{p}(n)=\varepsilon \& \mathrm{~d}(n)=\infty$ for any other node $n$

- $o q=\langle\mathrm{n} 2,2\rangle|\langle\mathrm{n} 1,6\rangle|\langle\mathrm{n} 3,6\rangle$

$\langle\mathrm{n} 2,2\rangle$ is the top element of $o q . \mathrm{n} 1$ and $\mathrm{n} 5$ are the direct successor nodes of $n 2$. Since $\mathrm{d}(\mathrm{n} 2)+\mathrm{w}(\mathrm{n} 2, \mathrm{n} 1)=3, \mathrm{~d}(\mathrm{n} 1)=4$ and $3<4, \mathrm{~d}(\mathrm{n} 1)$ is set to $3, \mathrm{p}(\mathrm{n} 1)$ is set to $(\mathrm{n} 0 \rightarrow \mathrm{n} 2 \rightarrow \mathrm{n} 1)$ and $\langle\mathrm{n} 1,5\rangle$, where $\mathrm{d}(\mathrm{n} 2)+\mathrm{w}(\mathrm{n} 2, \mathrm{n} 1)+\mathrm{h}(\mathrm{n} 1)=5$, is enqueued into oq. Since $\mathrm{d}(\mathrm{n} 2)+\mathrm{w}(\mathrm{n} 2, \mathrm{n} 5)=2, \mathrm{~d}(\mathrm{n} 5)=\infty$ and $2<\infty, \mathrm{d}(\mathrm{n} 5)$ is set to $2, \mathrm{p}(\mathrm{n} 5)$ is set to $(\mathrm{n} 0 \rightarrow \mathrm{n} 2 \rightarrow$ $\mathrm{n} 5)$ and $\langle\mathrm{n} 5,3\rangle$, where $\mathrm{d}(\mathrm{n} 2)+\mathrm{w}(\mathrm{n} 2, \mathrm{n} 5)+\mathrm{h}(\mathrm{b} 5)=3$, is enqueued into $o q$. The top element is deleted from $o q$. At this moment,

- $\mathrm{p}(\mathrm{n} 0)=\mathrm{n} 0 \& \mathrm{~d}(\mathrm{n} 0)=0$

- $\mathrm{p}(\mathrm{n} 1)=(\mathrm{n} 0 \rightarrow \mathrm{n} 2 \rightarrow \mathrm{n} 1) \& \mathrm{~d}(\mathrm{n} 1)=3$

- $\mathrm{p}(\mathrm{n} 2)=(\mathrm{n} 0 \rightarrow \mathrm{n} 2) \& \mathrm{~d}(\mathrm{n} 2)=1$

- $\mathrm{p}(\mathrm{n} 3)=(\mathrm{n} 0 \rightarrow \mathrm{n} 3) \& \mathrm{~d}(\mathrm{n} 3)=6$

- $\mathrm{p}(\mathrm{n} 4)=\varepsilon \& \mathrm{~d}(\mathrm{n} 4)=\infty$

- $\mathrm{p}(\mathrm{n} 5)=(\mathrm{n} 0 \rightarrow \mathrm{n} 2 \rightarrow \mathrm{n} 5) \& \mathrm{~d}(\mathrm{n} 5)=2$

- $o q=\langle\mathrm{n} 5,3\rangle|\langle\mathrm{n} 1,5\rangle|\langle\mathrm{n} 3,6\rangle$

$\langle\mathrm{n} 5,3\rangle$ is the top element of $o q . \mathrm{n} 1$ and $\mathrm{n} 3$ are the direct successor nodes of $n 5$. Since $\mathrm{d}(\mathrm{n} 5)+\mathrm{w}(\mathrm{n} 5, \mathrm{n} 1)=3, \mathrm{~d}(\mathrm{n} 1)=3$ and $3 \nless 3$, nothing changes. Since $\mathrm{d}(\mathrm{n} 5)+\mathrm{w}(\mathrm{n} 5, \mathrm{n} 3)=5$, $\mathrm{d}(\mathrm{n} 6)=6$ and $5<6, \mathrm{~d}(\mathrm{n} 3)$ is set to $5, \mathrm{p}(\mathrm{n} 5)$ is set to $(\mathrm{n} 0 \rightarrow \mathrm{n} 2 \rightarrow \mathrm{n} 5 \rightarrow \mathrm{n} 3)$ and $\langle\mathrm{n} 3,5\rangle$, where $\mathrm{d}(\mathrm{n} 5)+\mathrm{w}(\mathrm{n} 5, \mathrm{n} 3)+\mathrm{h}(\mathrm{n} 3)=5$, is enqueued into oq. The top element is deleted from oq. At this moment,

- $\mathrm{p}(\mathrm{n} 0)=\mathrm{n} 0 \& \mathrm{~d}(\mathrm{n} 0)=0$

- $\mathrm{p}(\mathrm{n} 1)=(\mathrm{n} 0 \rightarrow \mathrm{n} 2 \rightarrow \mathrm{n} 1) \& \mathrm{~d}(\mathrm{n} 1)=3$

- $\mathrm{p}(\mathrm{n} 2)=(\mathrm{n} 0 \rightarrow \mathrm{n} 2) \& \mathrm{~d}(\mathrm{n} 2)=1$

- $\mathrm{p}(\mathrm{n} 3)=(\mathrm{n} 0 \rightarrow \mathrm{n} 2 \rightarrow \mathrm{n} 5 \rightarrow \mathrm{n} 3) \& \mathrm{~d}(\mathrm{n} 3)=5$

- $\mathrm{p}(\mathrm{n} 4)=\varepsilon \& \mathrm{~d}(\mathrm{n} 4)=\infty$

- $\mathrm{p}(\mathrm{n} 5)=(\mathrm{n} 0 \rightarrow \mathrm{n} 2 \rightarrow \mathrm{n} 5) \& \mathrm{~d}(\mathrm{n} 5)=2$

- $o q=\langle\mathrm{n} 1,5\rangle \mid\langle\mathrm{n} 3,5\rangle$

$\langle\mathrm{n} 1,5\rangle$ is the top element of $o q . \mathrm{n} 4$ is the direct successor node of $\mathrm{n} 1$. Since $\mathrm{d}(\mathrm{n} 1)+\mathrm{w}(\mathrm{n} 1, \mathrm{n} 4)=4, \mathrm{~d}(\mathrm{n} 4)=\infty$ and $4<\infty, \mathrm{d}(\mathrm{n} 4)$ is set to $4, \mathrm{p}(\mathrm{n} 4)$ is set to $(\mathrm{n} 0 \rightarrow \mathrm{n} 2 \rightarrow \mathrm{n} 1 \rightarrow$ $\mathrm{n} 4)$ and $\langle\mathrm{n} 4,5\rangle$, where $\mathrm{d}(\mathrm{n} 1)+\mathrm{w}(\mathrm{n} 1, \mathrm{n} 4)+\mathrm{h}(\mathrm{n} 4)=5$, is enqueued into oq. The top element is deleted from oq. At this moment,

- $\mathrm{p}(\mathrm{n} 0)=\mathrm{n} 0 \& \mathrm{~d}(\mathrm{n} 0)=0$

- $\mathrm{p}(\mathrm{n} 1)=(\mathrm{n} 0 \rightarrow \mathrm{n} 2 \rightarrow \mathrm{n} 1) \& \mathrm{~d}(\mathrm{n} 1)=3$

- $\mathrm{p}(\mathrm{n} 2)=(\mathrm{n} 0 \rightarrow \mathrm{n} 2) \& \mathrm{~d}(\mathrm{n} 2)=1$

- $\mathrm{p}(\mathrm{n} 3)=(\mathrm{n} 0 \rightarrow \mathrm{n} 2 \rightarrow \mathrm{n} 5 \rightarrow \mathrm{n} 3) \& \mathrm{~d}(\mathrm{n} 3)=5$

- $\mathrm{p}(\mathrm{n} 4)=(\mathrm{n} 0 \rightarrow \mathrm{n} 2 \rightarrow \mathrm{n} 1 \rightarrow \mathrm{n} 4) \& \mathrm{~d}(\mathrm{n} 4)=4$

- $\mathrm{p}(\mathrm{n} 5)=(\mathrm{n} 0 \rightarrow \mathrm{n} 2 \rightarrow \mathrm{n} 5) \& \mathrm{~d}(\mathrm{n} 5)=2$

- $o q=\langle\mathrm{n} 3,5\rangle \mid\langle\mathrm{n} 4,5\rangle$

$\langle\mathrm{n} 3,5\rangle$ is the top element of oq. Since n3 is the goal node, we have found the path $\mathrm{n} 0 \rightarrow \mathrm{n} 2 \rightarrow \mathrm{n} 5 \rightarrow \mathrm{n} 3$ whose distance (or cost) is 5 . The path is one of the three shortest paths from n0 to $\mathrm{n} 3$ in DAG (1) shown in Fig. 1.
In this paper, we take into account a graph such that it is a DAG, a goal node is reachable from a start node and each edge is given a non-negative weight.

\section{FORMAL SPECIFICATION}

Let $K_{\mathrm{A} *}$ be the Kripke structure formalizing $\mathrm{A}^{*}$ that tackles a DAG in which there are $N$ nodes.

The four kinds of observable components are used:

- (node $[n i]: d, p s_{1}, p s_{2}, p$ ) - ni is a node ID, $d$ is a natural number or $\infty$ that is the distance of the path found so far from a start node to the node $n i, p s_{1} \& p s_{2}$ are soups of the direct successor node IDs of the node $n i$ that have not yet been tackled \& that have already been tackled and $p$ is the path found so far from a start node to the node $n i$; if $d$ is $\infty$, no path from a start node to the node $n i$ has yet been found;

- $(\circ q: q)-q$ is an ordered queue of node ID \& natural number pairs;

- (path: $n p p)$ - npp is a pair of a natural number and a path; when a path from a start node to a goal node is found, the pair of the distance of the path and the path is stored in it;

- (gstat: $g s$ ) - gs is either $\mathrm{nF}$ in or Fin; a path from a start node to a goal node has been found if $g s$ is $\mathrm{F}$ in.

Each state in $S_{\mathrm{A} *}$ is expressed as $\{o b s\}$, where obs is a soup of those observable components such that there is one gstat observable component, there is one path observable component, there is one oq observable component and there are $N$ node observable components.

$I_{\mathrm{A} *}$ consists of one state. When DAG (1) shown in Fig. 1 is tackled, the initial state is expressed as follows:

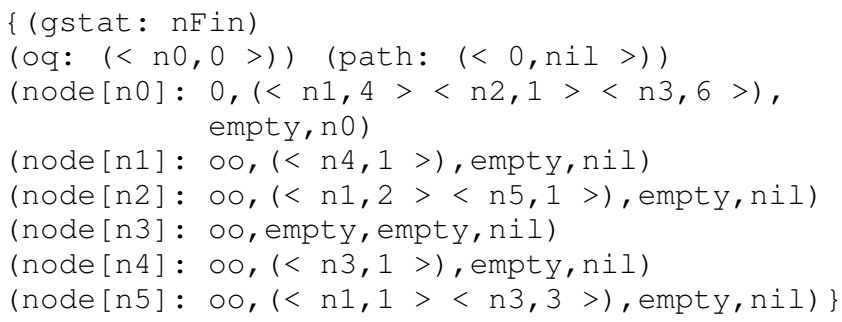

where $\langle\mathrm{n} 0, \mathrm{O}>$ in the oq observable component represents the singleton ordered queue that only consists of $<\mathrm{n} 0,0>$, nil is the empty path, empty is the empty soup, $\langle\mathrm{n} 1,4\rangle\langle\mathrm{n} 2,1\rangle\langle\mathrm{n} 3,6\rangle$ is the soup that consists of $<\mathrm{n} 1,4>,<\mathrm{n} 2,1>$ and $\langle\mathrm{n} 3,6>$, and ○ is $\infty$.

$T_{\mathrm{A} *}$ is specified as six rewrite rules. Let OCs be a Maude variable of observable component soups, NI \& NI' be Maude variables of node IDs, D, W, $D^{\prime}{ }^{\prime} \& D^{\prime} \prime^{\prime}$ be Maude variables of natural numbers, $D^{\prime}$ be a Maude variable of natural numbers or $\infty, Q$ be a Maude variable of ordered queues, NNPs1, NNPs2, NNPs1' \& NNPs2' be Maude variables of node ID \& natural number pair soups, and L, $L^{\prime} \& L^{\prime}$ be Maude variables of paths. 
The first one is as follows:

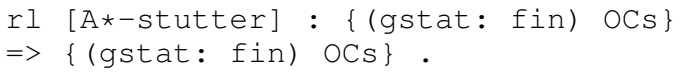

We need to use rule stutter to make $T_{\mathrm{A} *}$ total.

The second one is as follows:

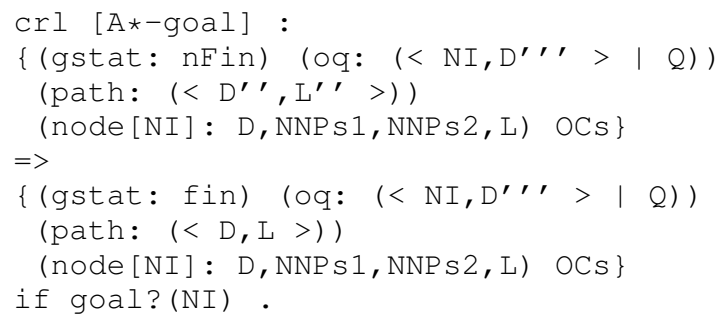

goal? (NI) holds if NI is the goal node ID. When the first element NI of the top element $<\mathrm{NI}, \mathrm{D}^{\prime}{ }^{\prime}{ }^{\prime}>$ of the ordered queue stored in the oq observable component is the goal node ID, then the path $\mathrm{L}$ has been found and $\langle\mathrm{D}, \mathrm{L}\rangle$, where $\mathrm{D}$ is its cost, is stored in the path observable component.

The third one is as follows:

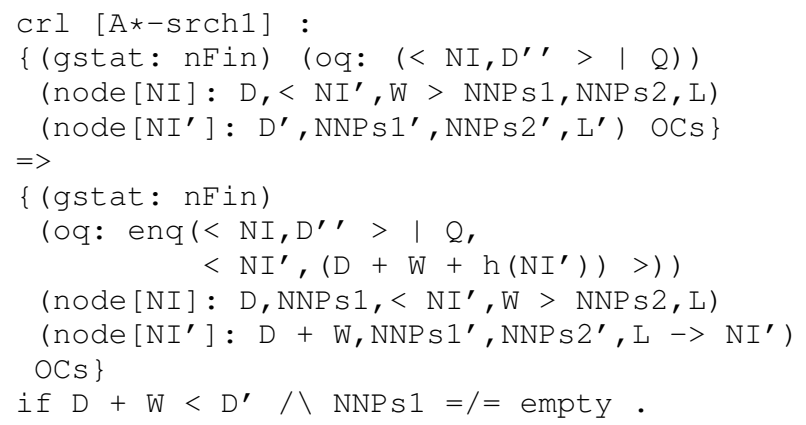

Rule srch1 says that if the gstate observable component is $\mathrm{nFin}$ (meaning that the path has not been found), NI is the node ID found in the top element pair of the ordered queue stored in the oq observable component, $\mathrm{NI}^{\prime}$ is a direct successor node ID such that it has not been tackled and the weight of the edge between $\mathrm{NI}$ and $\mathrm{NI}^{\prime}$ is $W, D+W$ is less than $D^{\prime}$ and NNPS 1 is not empty, then $<N I^{\prime},\left(D+W+h\left(N I^{\prime}\right)\right)>$ is put into the ordered queue stored in the oq observable component, $\left\langle\mathrm{NI}^{\prime}, \mathrm{W}\right\rangle$ is moved to the second soup from the first soup in the node [NI] observable component (meaning that NI' has been tackled), the path to $\mathrm{NI}^{\prime}$ from the start node is updated as $\mathrm{L} \rightarrow \mathrm{NI}$ ' obtained by adding $\mathrm{NI}^{\prime}$ to $\mathrm{L}$ at the end and the distance of the path is updated as $D+W$.

The fourth one is as follows:

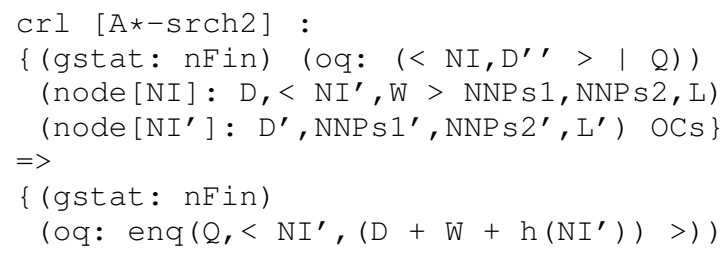

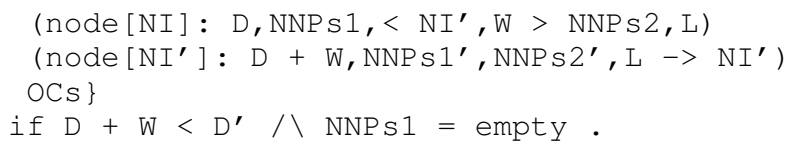

The only difference between the situations dealt with by rule srch1 and rule srch2 is whether NNPs1 is empty. If NNPS 1 is empty, the top element pair $\left\langle\mathrm{NI}, \mathrm{D}^{\prime}{ }^{\prime}>\right.$ is deleted from the ordered queue stored in the oq observable component.

The fifth one is as follows:

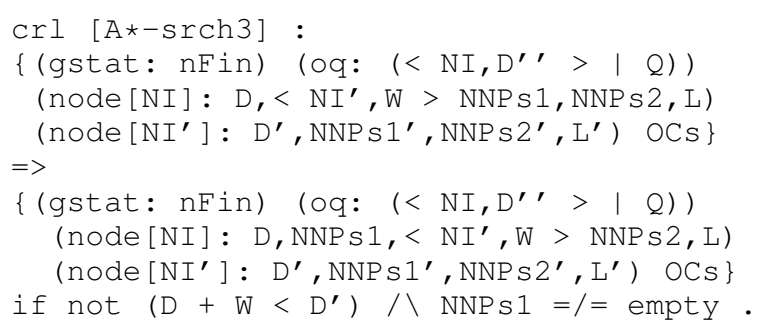

The only difference between the situations dealt with by rule $\operatorname{srch} 1$ and rule $\operatorname{srch} 3$ is whether $D+W<D^{\prime}$ holds. If $D+W<D^{\prime}$ does not, nothing changes except that $\left\langle\mathrm{NI}^{\prime}, \mathrm{W}\right\rangle$ is moved to the second soup from the first soup in the node [NI] observable component.

The sixth one $A *-\operatorname{srch} 4$ deals with the case in which $\mathrm{D}+\mathrm{W}<\mathrm{D}^{\prime}$ does not hold and NNPS1 is empty. If so, $<N I^{\prime}, W>$ is moved to the second soup from the first soup in the node [NI] observable component and the top element pair $<N I, D^{\prime}{ }^{\prime}>$ is deleted from the ordered queue stored in the oq observable component.

\section{Model Checking}

Let us consider a straightforward algorithm to find all paths from a start node to a goal node for a given graph. The algorithm is formalized as part of $K_{\text {all }}$ because we do not need to use $P_{\text {all }}$ and $L_{\text {all }}$.

The two kinds of observable components are used:

- (node $[n i]$ : nstat, $n, p s_{1}, p s_{2}, p s$ ) - ni is a node ID, nstat is a node status (which is one of notyet, visited and done), $n$ is the number of the incoming edges that have not been tackled, $p s_{1} \& p s_{2}$ are soups of the direct successor node IDs of the node $n i$ that have not yet been tackled \& that have already been tackled and $p s$ is a soup of $(d, p)$-pairs, where $p$ is a path from a start node to this node that has been found so far and $d$ is the distance; if nstat is notyet, the node has not been tackled, if it is visited, the node has been visited (partially tackled) and if it is done, the node has been fully tackled;

- (gstat $2: g s$ ) - gs is either $\mathrm{nF}$ in or $\mathrm{Fin}$; all paths from a start node to a goal node have been found if $g s$ is Fin.

$I_{\text {all }}$ consists of one state. When DAG (1) shown in Fig. 1 is tackled, the initial state is expressed as follows: 
$\{$ (gstat $2:$ nFin)

(node [n0]: done, $0,(<\mathrm{n} 1,4><\mathrm{n} 2,1><\mathrm{n} 3,6>$ ), empty, $<0, \mathrm{nO}>$ )

(node [n1]: notYet, 3, (< n 4, $1>$ ), empty, empty)

(node [n2] : notyet, $1,(<\mathrm{n} 1,2><\mathrm{n} 5,1>$ ), empty, empty)

(node [n3]: notYet, 3, empty, empty, empty)

(node $[n 4]$ : notyet, $1,(<\mathrm{n} 3,1>$ ), empty, empty)

(node [n5]: notYet, $1,(<\mathrm{n} 1,1><\mathrm{n} 3,3>$ ), empty, empty)\}

$T_{\text {all }}$ is specified as five rewrite rules. In addition to the Maude variables above-mentioned, let NLS \& NLS' be Maude variables of soups of (natural number,path)-pairs, $\mathrm{N}^{\prime}$ is a Maude variable of natural numbers and $N^{\prime}{ }^{\prime}$ is a Maude variable of node statuses. The first rule All-stutter is essentially the same as the one of $T_{\mathrm{A} *}$. The second one is as follows:

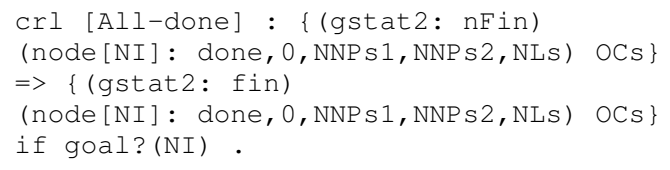

The rule says that if all paths to the goal node from the start node have been found, the value of the gstat 2 observable component is set to fin.

The last three rules are as follows:

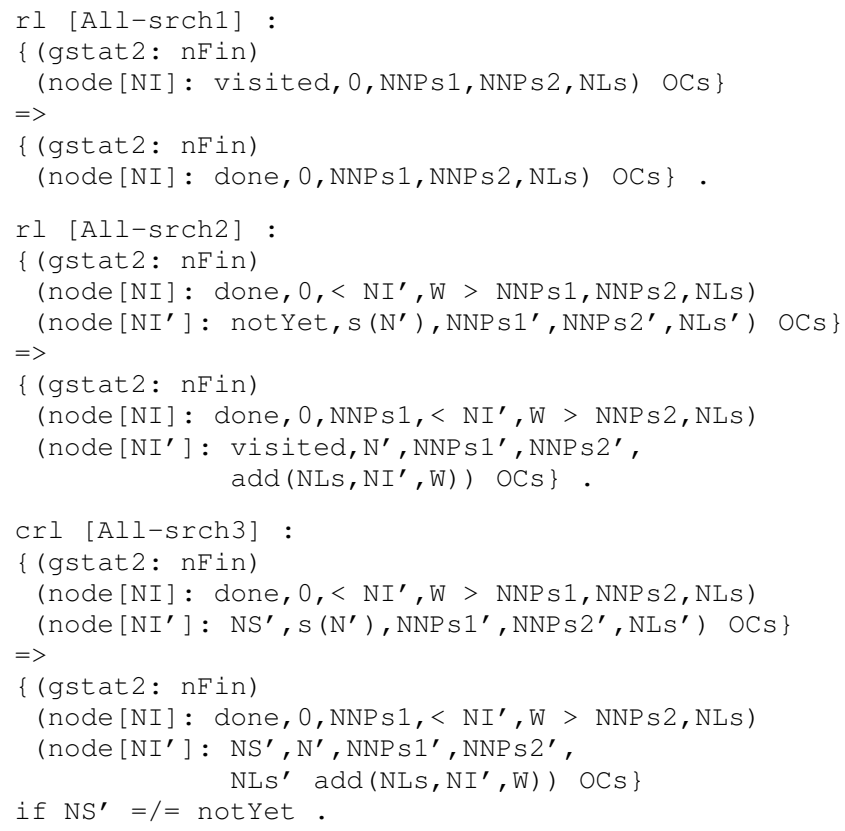

where $\mathrm{S}$ of $\mathrm{S}\left(\mathrm{N}^{\prime}\right)$ is the successor function of natural numbers.

If the first and second values of the node [NI] observable component are visited and 0 , the node NI has been fully tackled. If that is the case, the rule All-srchl changes the first value into done.

If the node NI has been fully tackled and has a direct successor $\mathrm{NI}^{\prime}$ that has not been treated and the first value of the node $\left[\mathrm{NI}^{\prime}\right]$ observable value is notYet, the first and second values of the node [NI'] observable value are changed into visited and the number $\mathrm{N}^{\prime}$ obtained by decrementing the original value $\mathrm{s}\left(\mathrm{N}^{\prime}\right)$; moreover, $\mathrm{NI}$, and $\mathrm{W}$ (which is the weight of the edge from NI to $\mathrm{NI}^{\prime}$ ) are added to each path in the soup NLs of the node [NI'] observable value. This is done by the rule $A l l-\operatorname{srch} 2$.

The rule $A l l-\operatorname{srch} 3$ does almost the same thing as the rule $A l l-\operatorname{srch} 2$. The difference is that the rule All-srch 3 deals with the case where the first value of the node $\left[\mathrm{NI}^{\prime}\right.$ ] observable value is not notYet, while the rule $A l l-\operatorname{srch} 2$ deals with the case where it is notyet.

The search command, where ALLPF is the specification of the straightforward algorithm and init is the initial state for ALLPF and the DAG shown in Fig 1,

search [1] in ALLPF : init

$=>\star\{$ (node $[\mathrm{n} 3]$ : done, 0, empty, NNPs2, NLs) OCs $\}$.

finds all paths to the node $\mathrm{n} 3$ from the node $\mathrm{n} 0$, which are assigned to NLS:

$\mathrm{NLS}-\rightarrow<5, \mathrm{n} 0->\mathrm{n} 2->\mathrm{n} 5->\mathrm{n} 3>$

$<5, \mathrm{n} 0->\mathrm{n} 2->\mathrm{n} 1 \rightarrow \mathrm{n} 4->\mathrm{n} 3>$

$<5, \mathrm{nO}->\mathrm{n} 2->\mathrm{n} 5 \rightarrow \mathrm{n} 1 \rightarrow \mathrm{n} 4->\mathrm{n} 3>$

$<6, \mathrm{nO}->\mathrm{n} 3><6, \mathrm{n} 0->\mathrm{n} 1->\mathrm{n} 4->\mathrm{n} 3>$

among which there are three shortest paths:

$<5, \mathrm{n} 0 \rightarrow \mathrm{n} 2 \rightarrow \mathrm{n} 5 \rightarrow \mathrm{n} 3>$

$<5, \mathrm{nO}->\mathrm{n} 2->\mathrm{n} 1 \rightarrow \mathrm{n} 4->\mathrm{n} 3>$

$<5, \mathrm{n} 0 \rightarrow \mathrm{n} 2 \rightarrow \mathrm{n} 5 \rightarrow \mathrm{n} 1 \rightarrow \mathrm{n} 4->\mathrm{n} 3>$

Let sPaths refer to the soup of the three shortest paths. The extraction of shortest paths from all paths to a goal node from a start node is done by a program written in Maude.

To model check that $K_{\mathrm{A} *}$ satisfies some desired properties, we define (or specify) $P_{\mathrm{A} *}$ and $L_{\mathrm{A} *}$. $P_{\mathrm{A} *}$ has two atomic propositions: $\mathrm{fin}$ and is SPath. $L_{\mathrm{A} *}$ is specified as follows:

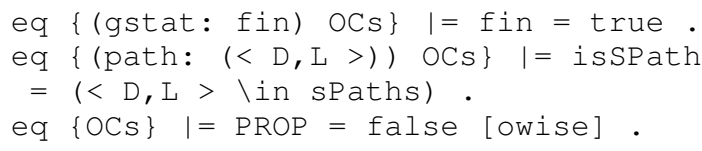

The three equations say that fin holds a state $s$ iff $s$ contains (gstat: fin) and issPath holds for a state $s$ iff $s$ contains (path: $(<\mathrm{D}, \mathrm{L}>)$ and sPaths contains $\langle\mathrm{D}, \mathrm{L}>$ as an element. Let two LTL formulas halt and correct be defined as $<>$ fin and [] (fin $-><>$ isSPath), where $<>$ is $\diamond$, [ ] is $\square$ and $->$ is $\Rightarrow$.

Let $\mathrm{h}$ be as follows: $\mathrm{h}(\mathrm{n} 1)=2, \mathrm{~h}(\mathrm{n} 3)=0$ and $\mathrm{h}(n)=1$ for any other node $n$, and init be the initial state for the specification ASTAR of $K_{\mathrm{A} *}$ and the DAG shown in Fig 1. We model check that $K_{\mathrm{A} *}$ satisfies halt and correct as follows:

red modelcheck (init, halt) . red modelcheck (init, correct). 


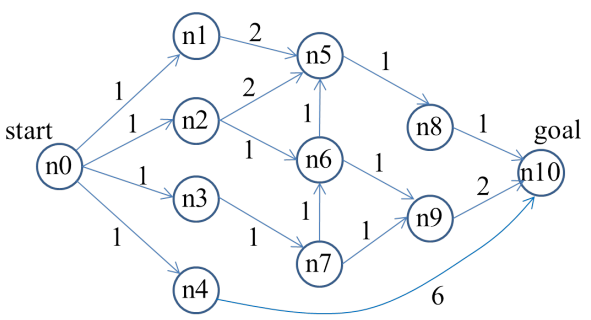

Figure 2. A DAG (2)

No counterexample is found for both model checking experiments. Because $h$ is admissible, namely that it never overestimates the actual cost to the goal $\mathrm{n} 3$ from any node, $K_{\mathrm{A} *}$ satisfies correct.

Let us use another $\mathrm{h}: \mathrm{h}(\mathrm{n} 1)=3, \mathrm{~h}(\mathrm{n} 5)=4, \mathrm{~h}(\mathrm{n} 3)=0$ and $\mathrm{h}(n)=1$ for any other node $n$. This $\mathrm{h}$ is not admissible because the actual cost to the goal $n 3$ from the node $n 1$ is 2 and the actual cost to the goal $n 3$ from the node $n 5$ is 3 . The first model checking experiment does not find any counterexamples, while the second one does. This is because $\mathrm{h}$ is not admissible. For this case, $K_{\mathrm{A} *}$ finds $\mathrm{n} 0 \rightarrow \mathrm{n} 3$, which is not the shortest one.

Let us use yet another $\mathrm{h}: \mathrm{h}(\mathrm{n} 1)=3, \mathrm{~h}(\mathrm{n} 3)=0$ and $h(n)=1$ for any other node $n$. This $h$ is not admissible either because the actual cost to the goal $n 3$ from the node $n 1$ is 2 . The two model checking experiments, however, do not find any counterexamples, meaning that $K_{\mathrm{A} *}$ still satisfies correct. In this case, $K_{\mathrm{A} *}$ finds n0 $\rightarrow$ n2 $\rightarrow$ n5 $\rightarrow$ n3. This is because for each node $n$ in the path, $\mathrm{h}(n)$ never overestimates the cost to the goal $\mathrm{n} 3$. This case may make us conjecture that if there exists a shortest path such that for each node $n$ in the path $\mathrm{h}(n)$ never overestimates the cost to the goal from $n$, then $\mathrm{A}^{*}$ finds a shortest path.

Let us consider the DAG shown in Fig. 2. Let us suppose that $\mathrm{h}$ is defined as follows: $\mathrm{h}(n)=0$ for all nodes $n$, namely that $A^{*}$ is equivalent to the Dijkstra algorithm. The two model checking experiments do not find any counterexamples.

Let us suppose that $\mathrm{h}$ is defined as follows: $\mathrm{h}(n)=10$ for all nodes $n$. This $\mathrm{h}$ is not admissible. Moreover, there exists no shortest path such that for each node $n$ in the path $\mathrm{h}(n)$ never overestimates the cost to the goal from $n$. The two model checking experiments, however, do not find any counterexamples. Thus, the sufficient condition that $A^{*}$ finds a shortest path can be relaxed.

A relaxed sufficient condition would be that there exists a shortest path such that for each node $n$ in the path $\mathrm{h}(n)$ plus the cost to $n$ from the start node is less than the cost of any non-shortest path to the goal from the start. Let us suppose that $\mathrm{h}(n 2)=5, \mathrm{~h}(n 4)=0, \mathrm{~h}(n 5)=3, \mathrm{~h}(n 6)=4$, $\mathrm{h}(n 4)=0, \mathrm{~h}(n 8)=2, \mathrm{~h}(n 10)=0$ and $\mathrm{h}(n)=10$ for any other node $n$. This $\mathrm{h}$ is not admissible but does satisfy the relaxed sufficient condition. The two model checking experiments do not find any counterexamples. Let us modify $\mathrm{h}$ a bit as follows: $\mathrm{h}(n 8)=2$ and for any other node $n$ $\mathrm{h}(n)$ is the same as the last version. This modified $\mathrm{h}$ does not satisfy the relaxed sufficient condition. The first model checking experiment does not find any counterexamples, while the second one finds a counterexample, where $A^{*}$ finds no $\rightarrow$ n $4 \rightarrow$ n10 that is not a shortest path. The two cases support that the relaxed sufficient condition is likely to make seance.

\section{CONCLUSION}

We have reported on a case study in which $\mathrm{A}^{*}$ algorithm is formally specified in Maude and model checked with the Maude LTL model checker. Two properties have been taken into account: the termination property and the correctness property. The former says whether $\mathrm{A}^{*}$ halts, while the latter says whether $A^{*}$ finds a shortest path. If the estimation $h$ is admissible, our model checking experiments say that $\mathrm{A}^{*}$ satisfies both properties. Our model checking experiments have also suggested that the condition can be relaxed. We have then conjectured that if there exists a shortest path such that for each node $n$ in the path $\mathrm{h}(n)$ plus the cost to $n$ from the start node is less than the cost of any non-shortest path to the goal from the start, $A^{*}$ finds a shortest path.

Among future directions are as follows. Many path finding algorithms have been proposed. One piece of our future work is to formally specify such algorithms in Maude and model check them with the Maude LTL model checker, coming up with a framework in which such algorithms can be systematically analyzed. Another piece of our future work is to apply Maude and the Maude LTL model checker to path planning for multiple robots or vehicles, which could contribute to a near future autonomous vehicle world.

\section{REFERENCES}

[1] P. E. Hart, et al., "A formal basis for the heuristic determination of minimum cost paths," IEEE Tans. Syst. Sci. Cybern., vol. 4, pp. 100-107, 1968.

[2] E. W. Dijkstra, "A note on two problems in connexion with graphs," Numerische Mathematik, vol. 1, pp. 269-271, 1959.

[3] M. Clavel, et al., All About Maude, ser. Lecture Notes in Computer Science. Springer, 2007, vol. 4350.

[4] A. K. Saberi, et al., "Analysis of path planning algorithms: a formal verification-based approach," in ECAL 2013, 2013, pp. 232-239.

[5] N. Maragos, et al., "A formal and executable model for path finding," in SEEFM 2003, 2003, pp. 129-139.

[6] A. Ulusoy, et al., "Optimality and robustness in multi-robot path planning with temporal logic constraints," I. J. Robotics Res., vol. 32, pp. 889-911, 2013. 